\title{
Prevalencia elevada de factores de riesgo cardiovascular en una población pediátrica
}

\author{
Germán Briceño, Mónica Fernández, Jaime Céspedes \\ Fundación Cardio-Infantil - Instituto de Cardiología, Bogotá, D.C., Colombia
}

Introducción. Los factores de riesgo cardiovascular pueden presentarse desde la infancia y predecir la enfermedad cardiovascular del adulto.

Objetivo. Determinar la prevalencia de los factores de riesgo cardiovascular de un grupo de niños, hijos de enfermeras de una institución de salud de Bogotá, Colombia.

Materiales y métodos. Se hizo un estudio de corte transversal en niños entre 3 y 17 años de edad, evaluados entre junio de 2011 y julio de 2012.

Resultados. Participaron 118 niños, con una edad promedio de 7,4 años (desviación estándar=3,86), la mayoría de ellos eutróficos $(72,0 \%)$. Los niños presentaban los siguientes factores de riesgo para enfermedad cardiovascular: malos hábitos alimenticios $(89,0 \%)$, sedentarismo $(78,8 \%)$, exposición al tabaco $(19,5 \%)$, historia familiar de riesgo cardiovascular $(16,1 \%)$, sobrepeso $(15,3 \%)$ y obesidad $(12,7 \%)$. No se encontraron diferencias estadísticamente significativas entre los factores de riesgo según sexo. El sedentarismo en niños con sobrepeso u obesidad fue de $90,9 \%$ y en niños eutróficos, de $36,5 \%$, p<0,001. En $84,8 \%$ de los niños con sobrepeso u obesidad y en $42,4 \%$ de los eutróficos, se presentaron malos hábitos alimentarios $(p<0,001)$. El $97,5 \%$ de los niños presentó, al menos, un factor de riesgo, y el $42,4 \%$, tres o más. La presencia de tres o más factores fue más frecuente en los obesos que en aquellos con sobrepeso $(p<0,001)$ y en los eutróficos $(p<0,001)$.

Conclusiones. Los resultados indican que los niños de 3 a 17 años incluidos en el estudio, presentaban varios factores de riesgo de enfermedad cardiovascular, en especial, aquellos con sobrepeso y obesidad.

Palabras clave: obesidad, sobrepeso, hipertensión, estilo de vida sedentario, hábitos alimentarios, enfermedades cardiovasculares.

doi: http://dx.doi.org/10.7705/biomedica.v35i2.2314

High prevalence of cardiovascular disease risk factors in a pediatric population

Introduction: Cardiovascular disease risk factors begin in childhood. Their presence may predict cardiovascular disease in adulthood.

Objective: To determine the prevalence of cardiovascular disease risk factors in a group of nurses' children at a health facility in Bogotá, Colombia.

Materials and methods: A cross-sectional, population-based observational study among 3-17 year-old individuals evaluated between June, 2011, and July, 2012.

Results: A total number of 118 children were included. The mean age was 7.4 years, with a standard deviation of $3.86 ; 72.0 \%$ of the children had a normal weight. Prevalence of cardiovascular risk factors was distributed as follows: inadequate food habits, $89.0 \%$; sedentary lifestyle, $78.8 \%$; family history of cardiovascular disease, $16.1 \%$; overweight, $15.3 \%$, and obesity, $12.7 \%$. There were no significant differences in risk factors between boys and girls. Among overweight and obese children, sedentary lifestyle was present in $90.9 \%$, and among normal-weight children, in $36.5 \%(p<0.001)$. Inadequate food habits were present in $84.8 \%$ of the overweight and obese children, and in $42.4 \%$ of those with normal weight $(p<0.001)$. Among the study population, $97.5 \%$ had at least one risk factor and $42.4 \%$ presented 3 or more. The presence of 3 or more cardiovascular disease risk factors was higher in obese children, when compared to overweight $(p<0.001)$ and normal-weight children $(p<0.001)$.

Conclusions: The results of this study indicate that there was a substantial burden of cardiovascular disease risk factors in the 3-17 year-old children included, particularly in those who were obese or overweight.

Key words: Obesity, overweight, hypertension, sedentary lifestyle, food habits, cardiovascular diseases. doi: http://dx.doi.org/10.7705/biomedica.v35i2.2314

\section{Contribución de los autores:}

Los tres autores intervinieron en todas las fases de la investigación. 
La enfermedad cardiovascular es la primera causa de muerte en el mundo (1) y, aunque sus manifestaciones son poco frecuentes durante la infancia y la adolescencia, la presencia de ciertos factores de riesgo (2) está asociada con la aparición de enfermedades crónicas en la vida adulta, incluidas la enfermedad aterosclerótica y la diabetes mellitus de tipo 2 (3-7).

La prevalencia de estos factores se ha incrementado en los últimos años en todo el mundo (4) y en Colombia, (8-10) donde se registra una prevalencia elevada de obesidad (13,71\%), sobrepeso $(32,7 \%)$, hipercolesterolemia total $(7,82 \%)$ e hipertensión arterial $(22,82 \%)$ en adultos, asociada con un riesgo de morir o enfermar de enfermedad cardiovascular en los siguientes 10 años cercano al $4 \%$ en hombres y mujeres entre los 50 y los 59 años de edad (9).

De igual manera, la prevalencia de obesidad $(5,2 \%)$ y de sobrepeso $(20,2 \%)$ en niños menores de 5 años, ha aumentado en el país en el último quinquenio (8). Sin embargo, no se encontraron estudios sobre los factores de riesgo de enfermedad cardiovascular en hijos menores de personas que laboren en el sector de la salud.

En Bogotá, la Fundación Cardio-Infantil - Instituto de Cardiología lidera un programa pediátrico de prevención de la enfermedad cardiovascular basado en el conocimiento de que algunos de los factores de riesgo asociados a su aparición se adquieren en edades muy tempranas en las que la prevención primaria es determinante (11-14). Por esta razón, se decidió llevar a cabo el presente estudio para conocer la prevalencia de los factores de riesgo de la enfermedad cardiovascular en niños de 3 a 17 años de edad hijos de enfermeras de la Fundación Cardio-Infantil, información que en el futuro permitirá realizar acciones en prevención primaria y secundaria.

\section{Materiales y métodos}

Entre junio de 2011 y julio de 2012, se hizo un estudio de corte transversal, con muestreo no probabilístico de casos consecutivos, el cual fue

\section{Correspondencia:}

Germán Darío Briceño, Carrera 13B N 163-85, torre A, piso 3, Bogotá, D.C., Colombia

Teléfono: (571) 667 2727, extensión 6300

dariobrice@gmail.com

Recibido: 27/02/14; aceptado: 05/02/15 aprobado por el Comité de Ética de la Fundación Cardio-Infantil y se ajustó a lo dispuesto en la Declaración del Helsinki (15).

El tamaño de la muestra se calculó en 111 niños bajo el supuesto de encontrar una prevalencia de sobrepeso del $15 \%$ (8) en la población de estudio, con una precisión de $6 \%$ y un nivel de confianza de $95 \%$.

Se invitó a participar en el estudio a los hijos entre 3 y 17 años de edad, de todas las enfermeras vinculadas a la Fundación Cardio-Infantil (N=394).

\section{Mediciones del estudio}

Las mediciones se hicieron de forma estandarizada, teniendo en cuenta los siguientes parámetros.

Talla. Se registró en centímetros, aproximando al $0,1 \mathrm{~cm}$ más cercano. Para la medición, se situó al niño descalzo sobre una superficie plana y horizontal, de espaldas al instrumento (tallímetro de madera, Hyssna, cinta de $215 \mathrm{~cm}$ ), con los pies paralelos y los talones, glúteos, hombros y cabeza en contacto con el plano posterior, con los brazos colgando a los lados de forma natural, manteniendo la línea horizontal imaginaria del ángulo externo del ojo y el conducto auditivo externo, o plano de Frankfort.

Peso. Se registró en kilogramos con una pesa de marca Soehnle de capacidad máxima de $150 \mathrm{~kg}$, calibrada cada cinco muestras con $1 \mathrm{~kg}$ de peso. Los niños debían estar descalzos y con el mínimo de ropa.

Índice de masa corporal. En este, también llamado índice de Quetelet, se calcula la relación entre el peso corporal en kilogramos sobre el cuadrado de la talla en metros. Los percentiles y desviaciones estándar correspondientes para la edad y el sexo, se evaluaron posteriormente.

Presión arterial. Para su medición, el niño requería un reposo mínimo de cinco minutos y no haber ingerido medicamentos o comidas estimulantes; se situaba con la espalda apoyada y los pies en contacto con el piso, el brazo derecho con soporte y la fosa cubital puesta a nivel del corazón. Se hicieron tres mediciones en cada ocasión, con manguitos de presión adecuados para cada edad, ubicados en un punto medio entre el acromion y el olécranon, mediante una técnica estandarizada (16).

Antecedentes personales. Se registraron los siguientes datos: hipertensión arterial del niño; consumo de medicamentos, como ácido retinoico, 
anticonceptivos orales o esteroides; enfermedades crónicas, como diabetes mellitus o síndrome nefrótico, y retardo en el crecimiento intrauterino definido como peso al nacer menor de $2.500 \mathrm{~g}$ en niños nacidos a término.

Antecedentes familiares. Se recopiló la información sobre los antecedentes de enfermedad cardiovascular temprana (menos de 55 años en hombres y menos de 65 años en mujeres) en familiares de primer y segundo grado de consanguinidad; de nivel de colesterol total de más de $240 \mathrm{mg} / \mathrm{dl}$ en alguno de los padres, de historia de diabetes mellitus en familiares de primer o segundo grado de consanguinidad.

Hábitos. Se evaluaron los hábitos alimentarios, la actividad física, el tabaquismo y el consumo de bebidas alcohólicas mediante un instrumento basado en la encuesta mundial de salud en escolares (Global School-based Student Health Survey, GSHS) (16). Los participantes de 9 años o más, de forma personal, y los menores, acompañados por sus padres, diligenciaron el cuestionario en un tiempo aproximado de 20 minutos. Se hicieron preguntas referentes al consumo de frutas, verduras y comida chatarra (junk food) en la última semana, incluidas las comidas principales y los refrigerios. Además, se indagó sobre la actividad física usual y en la semana previa a la valoración, así como sobre la práctica de actividades sedentarias.

\section{Definiciones del estudio}

Se identificaron los siguientes factores de riesgo, con base en las guías integradas de salud cardiovascular y reducción del riesgo en niños y adolescentes (2).

Obesidad y sobrepeso. Según los parámetros de los Centers for Disease Control and Prevention (CDC) de los Estados Unidos, un índice de masa corporal entre el percentil 85 y menos de 95, correspondió a sobrepeso y, uno de 95 o más, a obesidad (2). Además, se tuvieron en cuenta los parámetros de la Organización Mundial de la Salud (OMS), o sea, sobrepeso, con un índice de masa corporal entre 1 y 2 desviaciones estándar, y obesidad, con un índice de 2 o más desviaciones estándar (17).

Hipertensión arterial. Para su diagnóstico y clasificación, se tuvieron en cuenta la edad, el sexo y el percentil de talla. Una presión arterial, sistólica o diastólica, en el percentil entre 90 y menos de 95, según la edad, el sexo y la talla, se consideró como prehipertensión; lo mismo se aplicó para la tensión arterial de 120/80 o más mm de Hg, en adolescentes. La hipertensión arterial en estadio 1 se considera con valores entre el percentil 95 y 99 más $5 \mathrm{~mm}$ de $\mathrm{Hg}$, y en estadio 2, con valores por encima del percentil 99 más $5 \mathrm{~mm}$ de $\mathrm{Hg}$ (2).

Sedentarismo. Este se consideró presente en aquellos niños que no tenían actividad física (definida como cualquier actividad que acelerara los latidos del corazón y los dejara sin aliento en algunas ocasiones) durante al menos una hora al día, tres veces por semana, y que pasaran más de dos horas al día viendo televisión, navegando en internet o hablando por teléfono (2).

Malos hábitos alimentarios. Se los definió como una dieta sin suficientes frutas o verduras (tres o menos porciones al día) y que incluyera el consumo de fritos, postres o comida chatarra dos o más veces al día (18).

Exposición al tabaco. Se estableció como fumar cigarrillos en cualquier cantidad o frecuencia en cualquier momento de la vida, o como la exposición habitual al humo de cigarrillo (2).

Consumo de alcohol. Se lo consideró como el consumo de más de unos sorbos en cualquier momento de la vida.

Historia familiar de riesgo cardiovascular. Se consideró presente por el antecedente de enfermedad cardiovascular temprana, que incluía infarto agudo del miocardio, angina tratada, cateterismo coronario percutáneo, cirugía de derivación coronaria, muerte súbita o accidente cerebrovascular, en familiares de primer y segundo grado de consanguinidad (hombres menores de 55 años y mujeres menores de 65 años) (2).

Historia personal de riesgo cardiovascular. Esta se estableció como el antecedente de hipertensión arterial, de enfermedades crónicas, como diabetes mellitus o síndrome nefrótico, o de retardo del crecimiento intrauterino $(19,20)$.

\section{Análisis estadístico}

La prevalencia de las características demográficas y de las categorías nutricionales, se estimó con base en el índice de masa corporal (eutrófico, desnutrición, sobrepeso, obesidad), así como la frecuencia de factores de riesgo cardiovascular en la población de estudio (sedentarismo, malos hábitos alimentarios, exposición o consumo de tabaco, hipertensión arterial, historia personal o familiar de factores de riesgo de enfermedad cardiovascular). Además, se evaluaron la prevalencia y los intervalos de confianza de $95 \%$ de 
los factores de riesgo según sexo y grupo etario (preescolares, escolares y adolescentes), así como la distribución del número de factores de riesgo según las características demográficas (sexo, grupo etario, estado nutricional), evaluando las diferencias de prevalencia mediante la prueba de igualdad de proporciones con el paquete estadístico Stata 10.0.

\section{Resultados}

Entre junio de 2011 y julio de 2012, se valoraron 118 niños que aceptaron participar en el estudio en la Fundación Cardio-Infantil - Instituto de Cardiología. La edad promedio en años fue de 7,4 (desviación estándar $=3,86$ ), la mediana (percentiles de 25 a $75)$ fue de 7 (3,5 a 12,0), siendo ligeramente mayor en niños que en niñas. Con base en los parámetros de la OMS (17), se encontró $66,1 \%$ (78/118) de niños eutróficos, $22,9 \%(27 / 118)$ con sobrepeso, $6,8 \%(8 / 118)$ con obesidad y ninguno de bajo peso. Se encontraron proporciones similares con los parámetros de los CDC (2) (cuadro 1).

Los factores de riesgo más frecuentes fueron los malos hábitos alimentarios y el sedentarismo, seguidos por el exceso de peso (sobrepeso u obesidad), presente en $28,0 \%(33 / 118)$ de los niños (cuadro 2).

El sedentarismo en los niños con sobrepeso u obesidad, fue de $90,9 \%(30 / 33)$ y, en niños eutróficos, de $36,5 \%(31 / 85)(p<0,001)$. El 84,8\% (28/33) de aquellos con sobrepeso u obesidad tenía malos hábitos alimentarios, así como 42,4\% (36/85) de los eutróficos $(p<0,001)$.

No se encontraron diferencias clínicamente significativas en los factores de riesgo entre niños y niñas (cuadro 3). En cuanto al grupo etario, se encontró una alta prevalencia de sedentarismo en todas las edades, con diferencias estadísticamente significativas solamente entre preescolares y escolares $(p=0,02)$ (cuadro 4).

Casi toda la población de estudio, es decir, el 97,5\% $(115 / 118)$, presentó al menos un factor de riesgo $y$, el 42,4 \% (50/118), tres o más (cuadro 5). La presencia de tres o más factores de riesgo de enfermedad cardiovascular fue más frecuente en los niños obesos que en aquellos con sobrepeso $(p<0,001)$ o eutróficos $(p<0,001)$.

\section{Discusión}

Los resultados de este estudio muestran una alta prevalencia de factores de riesgo de enfermedad cardiovascular entre los niños evaluados de 3 a 17
Cuadro 1. Características demográficas y del estado nutricional de la población de estudio $(\mathrm{N}=118)$

\begin{tabular}{lr}
\hline Característica & $\mathbf{n}(\%)$ \\
\hline Sexo & \\
Femenino & $55(46,6)$ \\
Masculino & $63(53,4)$ \\
Edad (años) & \\
Preescolares: 2 a 5 & $44(37,3)$ \\
Escolares: 6 a 12 & $60(50,8)$ \\
Adolescentes: 13 a 18 & $14(11,9)$ \\
Estado nutricional * & \\
Bajo peso & $0(0,0)$ \\
Eutrofia & $85(72,0)$ \\
Sobrepeso & $18(15,3)$ \\
Obesidad & $15(12,7)$ \\
\hline
\end{tabular}

* Según parámetros de los Centers for Disease Control and Prevention

Cuadro 2. Prevalencia de factores de riesgo cardiovascular en la población de estudio $(\mathrm{N}=118)$

\begin{tabular}{|c|c|}
\hline Factor de riesgo & n (\%) \\
\hline Sobrepeso* & $18(15,3)$ \\
\hline Obesidad $^{*}$ & $15(12,7)$ \\
\hline Sedentarismo & $93(78,8)$ \\
\hline Malos hábitos alimentarios & $105(89,0)$ \\
\hline Exposición a tabaco & $23(19,5)$ \\
\hline Hipertensión arterial & $3(2,5)$ \\
\hline Historia personal de riesgo cardiovascular & $14(11,9)$ \\
\hline Antecedente de hipertensión arterial del niño & $0 \quad(0,0)$ \\
\hline Antecedente de enfermedades crónicas del niño & $0(0,0)$ \\
\hline $\begin{array}{l}\text { Antecedente de retardo del crecimiento } \\
\text { intrauterino del niño }\end{array}$ & $14(11,9)$ \\
\hline Historia familiar de riesgo cardiovascular & $19(16,1)$ \\
\hline Antecedente familiar de diabetes & $39(33,1)$ \\
\hline Antecedente familiar de hipercolesterolemia & $16(13,6)$ \\
\hline
\end{tabular}

* Según parámetros de los Centers for Disease Control and Prevention

años, presentándose al menos un factor de riesgo en $97,5 \%$ de ellos.

En cuanto al aspecto nutricional, la mayoría de los niños eran eutróficos. Sin embargo, la prevalencia de exceso de peso (sobrepeso y obesidad) en escolares fue mayor al descrito en estudios nacionales (8,21-23). Este hallazgo podría asociarse con el mayor nivel de sedentarismo encontrado en este grupo etario. En contraste, el exceso de peso en los adolescentes fue menor a lo reportado en la Encuesta Nacional de Situación Nutricional, ENSIN, 2010 (8).

Los resultados del estudio indican que los niños con sobrepeso y obesidad presentaron el mayor número de factores de riesgo de enfermedad cardiovascular $(86,7 \%$ con tres o más factores de riesgo), lo cual es similar a lo reportado en adolescentes de Estados Unidos (24) (cuadro 5). 
Cuadro 3. Prevalencia de factores de riesgo por sexo en niños de la población de estudio ( $N=118)$

\begin{tabular}{|c|c|c|c|c|}
\hline \multirow{3}{*}{ Factor de riesgo } & \multicolumn{4}{|c|}{ Sexo } \\
\hline & \multicolumn{2}{|c|}{ Femenino $n=55$} & \multicolumn{2}{|c|}{ Masculino $n=63$} \\
\hline & n (\%) & IC $_{95 \%}$ & n (\%) & $\mathrm{IC}_{95 \%}$ \\
\hline Sobrepeso* & $10(18,2)$ & $4,5-20,7$ & $8(12,7)$ & $4,2-21,1$ \\
\hline Obesidad $^{*}$ & $6(10,9)$ & $2,4-19,4$ & $9(14,3)$ & $5,4-23,1$ \\
\hline Sedentarismo & $46(83,6)$ & $73,5-93,7$ & $47(74,6)$ & $61,7-84,3$ \\
\hline Malos hábitos alimentarios & $49(89,1)$ & $78,2-94,9$ & $56(88,9)$ & $78,8-94,5$ \\
\hline Exposición a tabaco & $8(14,5)$ & $7,5-26,1$ & $15(23,8)$ & $15,0-35,6$ \\
\hline Hipertensión arterial & $1(1,8)$ & $0,31-9,6$ & $2(3,2)$ & $0,9-10,1$ \\
\hline Historia personal de riesgo cardiovascular & $8(14,5)$ & $7,5-26,1$ & $6 \quad(9,5)$ & $4,4-19,2$ \\
\hline Historia familiar de riesgo cardiovascular & $7(12,7)$ & $6,3-23,9$ & $12(19,0)$ & $9,1-29,0$ \\
\hline
\end{tabular}

* Según parámetros de los Centers for Disease Control and Prevention

Cuadro 4. Prevalencia de factores de riesgo en preescolares, escolares y adolescentes

\begin{tabular}{|c|c|c|c|c|c|c|}
\hline \multirow{3}{*}{ Factor de riesgo } & \multicolumn{6}{|c|}{ Grupo etario } \\
\hline & \multicolumn{2}{|c|}{ Preescolares $n=44$} & \multicolumn{2}{|c|}{ Escolares $n=60$} & \multicolumn{2}{|c|}{ Adolescentes $n=14$} \\
\hline & n (\%) & IC $_{95 \%}$ & n (\%) & IC $_{95 \%}$ & n (\%) & IC $_{95 \%}$ \\
\hline Sobrepeso* & $7(15,9)$ & $7,9-29,3$ & $9(15,0)$ & $5,7-24,3$ & $2(14,3)$ & $4,0-39,9$ \\
\hline Obesidad* & $5(11,4)$ & $5,0-24,0$ & $10(16,7)$ & $9,3-28,1$ & $0 \quad(0,0)$ & $0,0-21,5$ \\
\hline Sedentarismo & $31(70,5)$ & $55,8-81,9$ & $52(86,7)$ & $75,9-93,1$ & $10(71,4)$ & $45,3-88,3$ \\
\hline Malos hábitos alimentarios & $38(86,4)$ & $73,3-93,6$ & $53(88,3)$ & $77,8-94,2$ & $14(100,0)$ & $78,5-100,0$ \\
\hline Exposición a tabaco & $7(15,9)$ & $7,9-29,3$ & $11(18,3)$ & $10,5-29,9$ & $5(35,7)$ & $17,6-62,8$ \\
\hline Hipertensión arterial & $2(4,5)$ & $1,2-15,1$ & $1(1,7)$ & $0,3-8,9$ & $(0,0)$ & $0,0-21,5$ \\
\hline Historia personal de riesgo cardiovascular & $7(15,9)$ & $7,9-29,3$ & $7(11,6)$ & $5,7-22,1$ & $(0,0)$ & $0,0-21,5$ \\
\hline Historia familiar de riesgo cardiovascular & $7(15,9)$ & $7,9-29,3$ & $10(16,7)$ & $9,3-28,1$ & $2(14,3)$ & $4,0-39,9$ \\
\hline
\end{tabular}

* Según parámetros de los Centers for Disease Control and Prevention

Cuadro 5. Distribución del número de factores de riesgo según características demográficas

\begin{tabular}{|c|c|c|c|c|c|}
\hline \multirow{2}{*}{\multicolumn{2}{|c|}{ Característica demográfica }} & \multicolumn{4}{|c|}{ Número de factores de riesgo } \\
\hline & & $0(n=3)$ & $\begin{array}{c}1(n=20) \\
n(\%)\end{array}$ & $\begin{array}{c}2(n=45) \\
n(\%)\end{array}$ & $\begin{array}{c}\geq 3(n=50) \\
n(\%)\end{array}$ \\
\hline \multicolumn{6}{|l|}{ Sexo } \\
\hline Femenino & $(n=55)$ & $0(0,0)$ & $10(18,2)$ & $24(43,6)$ & $21(38,2)$ \\
\hline Masculino & $(n=63)$ & $3(4,8)$ & $10(15,9)$ & $21(33,3)$ & $29(46,0)$ \\
\hline \multicolumn{6}{|l|}{ Grupo etario } \\
\hline Preescolares & $(n=44)$ & $3(6,8)$ & $8(18,2)$ & $16(36,4)$ & $17(38,6)$ \\
\hline Escolares & $(n=60)$ & $0(0,0)$ & $9(15,0)$ & $25(41,7)$ & $26(43,3)$ \\
\hline Adolescentes & $(n=14)$ & $0(0,0)$ & $3(21,4)$ & $4(28,6)$ & $7(50,0)$ \\
\hline \multicolumn{6}{|c|}{ Estado nutricional ${ }^{*}$} \\
\hline Eutrofia & $(n=85)$ & $3(3,5)$ & $19(22,4)$ & $40(47,1)$ & $23(27,1)$ \\
\hline Sobrepeso & $(n=18)$ & $0(0,0)$ & $0(0,0)$ & $4(22,2)$ & $14(77,8)$ \\
\hline Obesidad & $(n=15)$ & $0(0,0)$ & $1 \quad(6,7)$ & $1(6,7)$ & $13(86,7)$ \\
\hline
\end{tabular}

* Según parámetros de los Centers for Disease Control and Prevention

El $36,5 \%$ de los niños eutróficos eran sedentarios y el 42,4\% no tenía una alimentación saludable, lo que representa una oportunidad para la implementación de programas que promuevan estilos de vida saludables, mejorando su alimentación en el hogar y en la escuela, independientemente de su estado nutricional.
La prevalencia del sedentarismo en adolescentes fue menor a la encontrada previamente en un estudio de niños de 13 a 15 años en Bogotá (83,4\%) (25). En un estudio en Medellín (21), el grupo de escolares y el de niñas presentaron una mayor proporción de sedentarismo, en comparación con los preescolares y adolescentes (cuadro 4). 
En este estudio, la proporción de niños y adolescentes que habían tenido actividad física, por lo menos, 60 minutos al día durante la semana anterior a la evaluación, fue mayor $(21,2 \%)$ que la reportada previamente en Bogotá (15\%) (26), condición que puede modificarse adoptando políticas escolares que promuevan la actividad física.

La exposición al tabaco encontrada fue de 19,5\%, siendo más frecuente en niños que en niñas, tal como se ha concluido en estudios locales (22). Aunque una mayor proporción de los adolescentes estuvo expuesta al tabaco y a malos hábitos alimentarios, en comparación con los preescolares y escolares, el escaso número de adolescentes participantes no permitió evaluar la significancia estadística. Los adolescentes viven cambios emocionales y sociales importantes, y están sujetos a presiones de grupo y a la búsqueda de independencia, lo que podría explicar su mayor consumo de comidas rápidas, así como la elección inapropiada de refrigerios y un deficiente cuidado personal.

El $42,4 \%$ de la población tuvo tres o más factores de riesgo de enfermedad cardiovascular, con diferencias según las características demográficas, siendo los niños, los obesos y los adolescentes los grupos de población más afectados (cuadro 5). Los hallazgos sugieren que cuando se presenta, al menos, un factor de riesgo de enfermedad cardiovascular, hay mayor frecuencia de factores adicionales, tal como se encontró en el estudio Bogalusa $(27,28)$.

Llamó la atención que esta población de hijos de enfermeras de una institución dedicada a la atención en salud cardiovascular, presentara una frecuencia de factores de riesgo cardiovascular modificables similar a la de la población pediátrica general y, en algunos casos, mayor. Aunque esto podría deberse a un sesgo de selección de la población de estudio, pareciera que una de las mayores dificultades culturales tiene que ver con la coherencia entre el conocimiento de aquello que debe hacerse para promover la salud (deber ser) $y$ aquello que efectivamente se hace en la vida cotidiana. Dicha incoherencia podría responder, además, a la poca atención que los profesionales, incluidos los de la salud, prestan a ciertos aspectos que influyen en el cuidado personal, como, por ejemplo, el tiempo de descanso o de ocio, el tiempo de recreación en compañía de los hijos, los espacios para compartir en familia, el tiempo para hacer ejercicio, para hacer un mercado y cocinar comida saludable, el espacio para soñar y pensar en el bienestar propio, así como las relaciones en la familia. Estos factores podrían atribuirse a aspectos relacionados con la economía; sin embargo, desde una perspectiva ecológica, podría cuestionarse el hecho de que, con el mismo tiempo, espacio y recursos, sea posible organizar la vida de tal manera que se brinde la atención necesaria a la salud. Por otro lado, el personal que labora en el sector de la salud tendría que liderar la implementación de políticas y estrategias de promoción de ambientes saludables, para prevenir factores de riesgo entre sus hijos (prevención primordial).

Otra limitación del estudio fue el reducido número de adolescentes incluidos, lo que disminuye el poder para identificar diferencias, en caso de que existieran. Además, no se hicieron pruebas de glucemia, colesterol, triglicéridos y perímetro abdominal como indicador de obesidad central, las cuales podrían haber brindado información adicional sobre el riesgo cardiovascular. Los resultados del estudio reflejan la situación de un grupo de niños hijos de enfermeras y no pretenden ser extrapolables directamente a otra población.

Los resultados sugieren que la estrategia de tamización de los factores de riesgo de la enfermedad cardiovascular en niños, es un método eficiente para su identificación y sirve de punto de partida de acciones de prevención primaria y secundaria de la enfermedad cardiovascular en la vida adulta (11). De igual forma, resaltan la importancia de la continuidad de los programas locales $(29)$ y nacionales $(12,30)$ que promueven la actividad física y una alimentación saludable.

La promoción de estilos de vida saludables debe involucrar a los prestadores de servicios de salud, así como la tamización oportuna de los factores de riesgo cardiovascular y la participación de otros actores, entre ellos la familia, la comunidad y los medios de comunicación, que deben recibir educación sobre la alimentación saludable y la actividad física. Igualmente, deben adoptarse políticas locales y nacionales que favorezcan los estilos de vida saludables orientados a garantizar una salud cardiovascular ideal.

Las escuelas y los programas de salud escolar son componentes centrales de las estrategias comunitarias de prevención primaria de la enfermedad cardiovascular desde la infancia (31). En nuestro medio, se han hecho intervenciones en las instituciones educativas como instrumento para lograr cambios en las actitudes, conocimientos y hábitos de los preescolares y de sus padres, que 
fomenten un estilo de vida activo y una alimentación saludable, cambios que perduran incluso hasta tres años después de finalizada la intervención $(13,14)$.

En conclusión, los niños de la población de estudio presentaron una elevada prevalencia de factores de riesgo cardiovascular, en especial, aquellos con sobrepeso y obesidad, lo que resalta la importancia de la tamización de los factores de riesgo de la enfermedad cardiovascular desde el nacimiento, así como de la educación del niño y su familia, como instrumentos de prevención primaria. Por otra parte, es esencial propender por espacios laborales que estimulen un estilo de vida saludable, lo que a su vez promueve la salud cardiovascular ideal en las familias.

A partir de la información obtenida con los instrumentos de tamización descritos, se hará el seguimiento de los pacientes en quienes se identificaron factores de riesgo de enfermedad cardiovascular, sensibilizando a los niños y a sus familias en torno a la importancia del reconocimiento de estos factores y su manejo.

En estudios futuros se deberá evaluar también la salud cardiovascular ideal de la población pediátrica y de las familias, utilizando acelerómetros como herramientas de medición precisa de la actividad física.

\section{Conflicto de intereses}

Los autores declaran no tener conflictos de intereses en relación con este artículo.

\section{Financiación}

El estudio fue financiado por el Departamento de Investigaciones de la Fundación Cardio-Infantil Instituto de Cardiología.

\section{Referencias}

1. World Health Organization. World Health Report 2002. Reducing Risks, Promoting Healthy Life. Geneva: WHO; 2002. Fecha de consulta: 9 de noviembre de 2012. Disponible en: http://www.who.int/whr/2002/en/whr02_en. pdf?ua $=1$.

2. Expert Panel on Integrated Guidelines for Cardiovascular Health and Risk Reduction in Children and Adolescents. Expert Panel on Integrated Guidelines for Cardiovascular Health and Risk Reduction in Children and Adolescents: Summary Report. Pediatrics. 2011;128(Suppl.5):S213-56. http://dx.doi.org/10.1542/peds.2009-2107C

3. Singh AS, Mulder C, Twisk JW, van Mechelen W, Chinapaw MJ. Tracking of childhood overweight into adulthood: A systematic review of the literature. Obes Rev. 2008;9:474-88. http://dx.doi.org/10.1111/j.1467-789X. 2008.00475.x
4. Lloyd LJ, Langley-Evans SC, McMullen S. Childhood obesity and risk of the adult metabolic syndrome: A systematic review. Int J Obes (Lond). 2012;36:1-11. http:// dx.doi.org/10.1038/ijo.2011.186

5. Craigie A, Lake A, Kelly S, Adamson A, Mathers J. Tracking of obesity-related behaviours from childhood to adulthood: A systematic review. Maturitas. 2011;70:266-84. http://dx.doi.org/10.1016/j.maturitas.2011.08.005

6. Strong JP, Malcom GT, McMahan CA, Tracy RE, Newman WP III, Herderick EE, et al. Prevalence and extent of atherosclerosis in adolescents and young adults: Implications for prevention from the Pathobiological Determinants of Atherosclerosis in Youth Study. JAMA. 1999;281:727-35. http://dx.doi.org/10.1001/jama.281.8.727

7. Magnussen CG, Koskinen J, Chen W, Thomson R, Schmidt MD, Srinivasan SR, et al. Pediatric metabolic syndrome predicts adulthood metabolic syndrome, subclinical atherosclerosis, and type 2 diabetes mellitus but is no better than body mass index alone: The Bogalusa Heart Study and the Cardiovascular Risk in Young Finns Study. Circulation. 2010;122:1604-11. http://dx.doi.org/10.1161/ CIRCULATIONAHA.110.940809

8. Ministerio de la Protección Social. Encuesta Nacional de la Situación Nutricional en Colombia (ENSIN) 2010. Fecha de consulta: 15 de agosto de 2012. Disponible en: http://www.icbf.gov.co/portal/page/portal/Descargas1/ Resumenfi.pdf.

9. Ministerio de la Protección Social. Encuesta Nacional de Salud (ENS) 2007. Fecha de consulta: 15 de agosto de 2012. Disponible en: http://www.minsalud.gov.co/ Documentos\%20y\%20Publicaciones/ENCUESTA\%20 NACIONAL.pdf.

10. Villarreal E, Forero Y, Poveda E, Baracaldo C, López E. Marcadores de riesgo cardiovascular en escolares de cinco departamentos de la región oriental en Colombia. Biomédica. 2008;28:38-49. http://dx.doi.org/10.7705/ biomedica.v28i1.107

11. Laitinen T, Pahkala K, Magnussen C, Viikari JS, Oikonen M, Taittonen L, et al. Ideal cardiovascular health in childhood and cardiometabolic outcomes in adulthood: The Cardiovascular Risk in Young Finns Study. Circulation. 2012;125:1971-8. http://dx.doi.org/10.1161/ CIRCULATIONAHA.111.073585

12. Céspedes J, Briceño G, Farkouh ME, Vedanthan R, Baxter $\mathbf{J}$, Leal $\mathbf{M}$, et al. Targeting preschool children to promote cardiovascular health: Cluster randomized trial. Am J Med. 2013;126:27-35. http://dx.doi.org/10.1016/j. amjmed.2012.04.045

13. Céspedes J, Briceño G, Farkouh $\mathbf{M}$, Vedanthan $\mathbf{R}$, Baxter J, Leal M, et al. Promotion of cardiovascular health in preschool children: 36-month cohort follow-up. Am J Med. 2013;126:1122-6. http://dx.doi.org/10.1016/j. amjmed.2013.06.021

14. Céspedes J, Briceño G, Farkouh M, Vedanthan R, Leal $\mathbf{M}$, Dennis $\mathbf{R}$, et al. A randomized preschool trial to promote cardiovascular health in Colombia: 18 month-follow up. Circulation. 2012;125:AP186.

15. Asociación Médica Mundial. Declaración de Helsinki: Principios éticos para las investigaciones médicas en seres humanos, 2013. Fecha de consulta: 11 de 
noviembre de 2013. Disponible en: http://www.wma.net/ es/30publications/10policies/b3/index.html.pdf?printmedia-type\&footer-right=[page]/[toPage].

16. World Health Organization. Chronic diseases and health promotion: Global school-based student health survey (GSHS): 2009. Fecha de consulta: 22 de junio de 2010. Disponible en: http://www.who.int/chp/gshs/methodology/en/.

17. World Health Organization. Child growth standards: Length/height-for-age, weight-for-age, weight-for-length, weight-for-height and body mass index-for-age - 2006 . Fecha de consulta: 3 de abril de 2010. Disponible en: http:// www.who.int/childgrowth/standards/technical_report/en/.

18. World Health Organization. Dietary intake of fruits and vegetables and management of body weight, 2004. Fecha de consulta: 22 de junio de 2010. Disponible en: http:// www.who.int/dietphysicalactivity/publications/f\&v_weight_ management.pdf.

19. Varvarigou AA. Intrauterine growth restriction as a potential risk factor for disease onset in adulthood. J Pediatr Endocrinol Metab. 2010;23:215-24.

20. Meas T, Deghmoun S, Armoogum P, Alberti C, LevyMarchal C. Consequences of being born small for gestational age on body composition: An 8-year follow-up study. J Clin Endocrinol Metab. 2008;93:3804-9. http:// dx.doi.org/10.1210/jc.2008-0488

21. Uscátegui RM, Álvarez MC, Laguado W, Soler L, Martínez $\mathbf{L}$, Arias $\mathbf{R}$, et al. Factores de riesgo cardiovascular en niños de 6 a 18 años de Medellín (Colombia). An Pediatr. 2003;58:411-7. http://dx.doi.org/10.1016/S16954033(03)78086-2

22. Durán AE, Martínez MP, Rodríguez DC, Mosquera W, Villa-Roel C. Estudio del riesgo cardiovascular en la infancia a través de un modelo clínico-investigativo. Rev Col Cardiol. 2006;13:128-31.

23. Gracia B, de Plata C, Méndez F, Cruz M, Leiva J, Conde L, et al. Evaluación de manifestaciones tempranas de riesgo para enfermedades crónicas no trasmisibles en población escolarizada de Cali, Colombia. ALAN. 2005;55:267-78.
24. May A, Kuklina E, Yoon P. Prevalence of cardiovascular disease risk factors among US adolescents, 1999-2008. Pediatrics. 2012;129:1035-41. http://dx.doi.org/10.1542/ peds.2011-1082

25. Piñeros M, Pardo C. Actividad física en adolescentes de cinco ciudades colombianas: resultados de la Encuesta Mundial de Salud a Escolares. Rev Salud Pública. 2010;12: 903-14. http://dx.doi.org/10.1590/S0124-00642010000600003

26. Instituto Nacional de Cancerología. Encuesta Mundial de Salud a Escolares - Global School-based Student Health Survey. Resumen informativo. Bogotá, D.C: INC; 2007. Fecha de consulta: 8 de mayo de 2012. Disponible en: http://www.cancer.gov.co/documentos/EncuestaEMSE/ ResumenBogota.pdf.

27. Berenson GS, Srinivasan SR, Bao W, Newman WP III, Tracy RE, Wattigney WA. Association between multiple cardiovascular risk factors and atherosclerosis in children and young adults: The Bogalusa Heart Study. N Engl J Med. 1998;338:1650-6. http://dx.doi.org/10.1056/ NEJM199806043382302

28. Andersen LB, Wedderkopp N, Hansen HS, Cooper AR, Froberg K. Biological cardiovascular risk factors cluster in Danish children and adolescents: The European Youth Heart Study. Prev Med. 2003;37:363-7. http://dx.doi.org/10.1016/ S0091-7435(03)00145-2

29. Secretaría de Cultura, Recreación y Deporte, Alcaldía Mayor de Bogotá, D. C. Bogotá más activa. Fecha de consulta: 8 de mayo de 2012. Disponible en: http://www. culturarecreacionydeporte.gov.co/areas-de-trabajo/ actividad-fisica-y-deporte.

30. Instituto Colombiano del Deporte. Programa Nacional de Actividad Física: Colombia Activa y Saludable. Fecha de consulta: 8 de mayo de 2012. Disponible en: http://www.coldeportes.gov.co/coldeportes/index.php? idcategoria $=6301$

31. Hoehner C. Physical activity interventions in Latin America: A systematic review. Am J Prev Med. 2008;34:224-33. ttp:// dx.doi.org/10.1016/j.amepre.2007.11.016 